\title{
Langzeitergebnisse nach Stripping, EVLA und Schaumsklerosierung
}

Vähäaho S et al. Five-year follow-up of a randomized clinical trial comparing open surgery, foam sclerotherapy and endovenous laser ablation for great saphenous varicose veins. BJS. 2018;105:686-691. Doi:10.1002/bjs.1075

Die finnische Arbeitsgruppe hatte bereits die 1-Jahres-Ergebnisse ihrer randomisierten Studie über die Refluxbehandlung der V. saphena magna (GSV) vorgestellt. Insgesamt 214 Patienten im Alter von 20-70 Jahren mit einer Varikosis (CEAP C2-C4) nahmen an der Studie teil. Nach Stripping, endovenöser Laserablation (EVLA) oder Schaumsklerosierung (UGFS) wiesen in der UGFS-Gruppe mehr Patienten Rekanalisationen und einen erneuten Reflux auf. In den beiden anderen Gruppen waren die Erfolgsraten hoch und nicht signifikant verschieden. In der subjektiven Einschätzung der Patienten ergaben sich keine Gruppenunterschiede bzgl. der krankheitsassoziierten Lebensqualität. 166 Patienten nahmen an der 5-Jahre-Untersuchung teil (50 nach Stripping, 57 nach EVLA und 59 nach UGFS). Das Assessment schloss eine Dopp- lersonografie, klinische Untersuchung und die Messung der Lebensqualität ein (Aberdeen Varicose Vein Severity Score AVVSS).

Ein vollständiger Verschluss der GSV nach 5 Jahren bestand bei $96 \%$ (Stripping), 89\% (EVLA) und $51 \%$ (UGFS). Für den AVVSS ergaben sich keine Gruppenunterschiede, wobei die UGFS tendenziell besser abschnitt. 22 Patienten hatten bereits eine zusätzliche Therapie erhalten. Dies waren nach einem Stripping 4\%, nach EVLA $0 \%$ und nach UGFS $34 \%$. Die Subanalyse auf nicht-assistierte Okklusionen reduzierte die Verschlussrate nach UGFS auf $41 \%$. Die Gesamtwahrscheinlichkeit für eine erneute Behandlung war verglichen mit der EVLA für die UGFS 8,5-fach gesteigert. Eine 2. Therapie kam nach EVL 1,6-mal öfter vor als nach der Operation. Schwerwiegende Neben- wirkungen traten nicht auf. Hautpigmentierungen bestanden bei $4 \%$ (Stripping), $2 \%$ (EVLA) und $4 \%$ (UGFS). In jeder Gruppe empfand jeweils 1 Patient eine Hypästhesie. Bei 1 Patienten war nach dem Stripping eine Hautschwellung tastbar.

Die UGFS sei zwar die kurzfristig kostengünstigste, aber langfristig ineffektivste Therapievariante, so die Autoren. Nur $27 \%$ der UGFS-Gruppe bedurften keiner weiteren Intervention. Möglicherweise verbesserten häufigere Nachsorgen und Resklerosierungen die Langzeitresultate. Die Autoren empfehlen die UGFS für Rezidive und Seitenastvarikosen. Stripping und EVLA seien die besten Methoden bei einem primären Reflux der V. saphena magna.

Dr. med. Susanne Krome, Melle 